\title{
Endoscopic ultrasound-guided fine needle injection of alcohol for ablation of an insulinoma: a well documented successful procedure
}

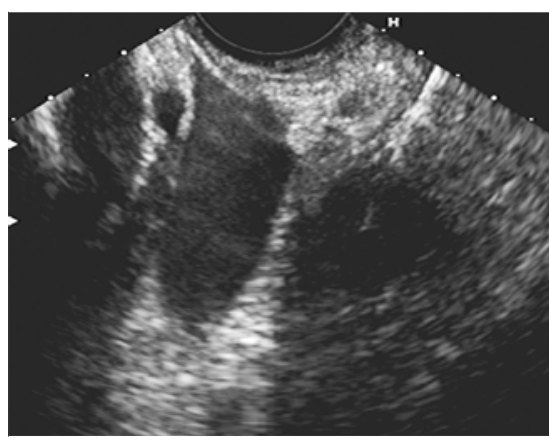

- Fig. 1 Echoendoscopic image showing the endoscopic ultrasound-guided fine needle aspiration.

A 21-year-old woman presented with recurrent hypoglycemia, syncope, and episodic seizures, associated with rapid weight gain (body mass index [BMI] 60). Hyperinsulinemia was confirmed and she was started on treatment with diazoxide. Magnetic resonance imaging showed a $1.5-\mathrm{cm}$ nodule at the pancreatic head. Endoscopic ultrasound (EUS) confirmed a $1.5-\mathrm{cm}$ hyperechoic nodule within the pancreatic head, between the common bile duct and the main pancreatic duct. EUS-guided fine needle aspiration (EUSFNA) was performed with a 22-gauge needle (Echo-Tip; Cook Medical, Limerick, Ireland) ( $\triangleright$ Fig. 1) and the definitive diagnosis of an insulinoma was established by immunohistochemical analysis ( Fig. 2).

Surgical treatment (Whipple's procedure) was contraindicated because of the patient's high BMI. Therefore, EUSguided fine needle injection (EUS-FNI) was suggested to ablate the lesion. The patient was hospitalized for prior hyperhydration. EUS-FNI was performed with $99 \%$ ethanol $(1.5 \mathrm{~mL})$ using a 22 -gauge needle (Echo-Tip), with the patient under general anesthesia. After 60 seconds, ultrasound monitoring showed the typical image of a hyperechogenic shadow forming from within the lesion. No portal

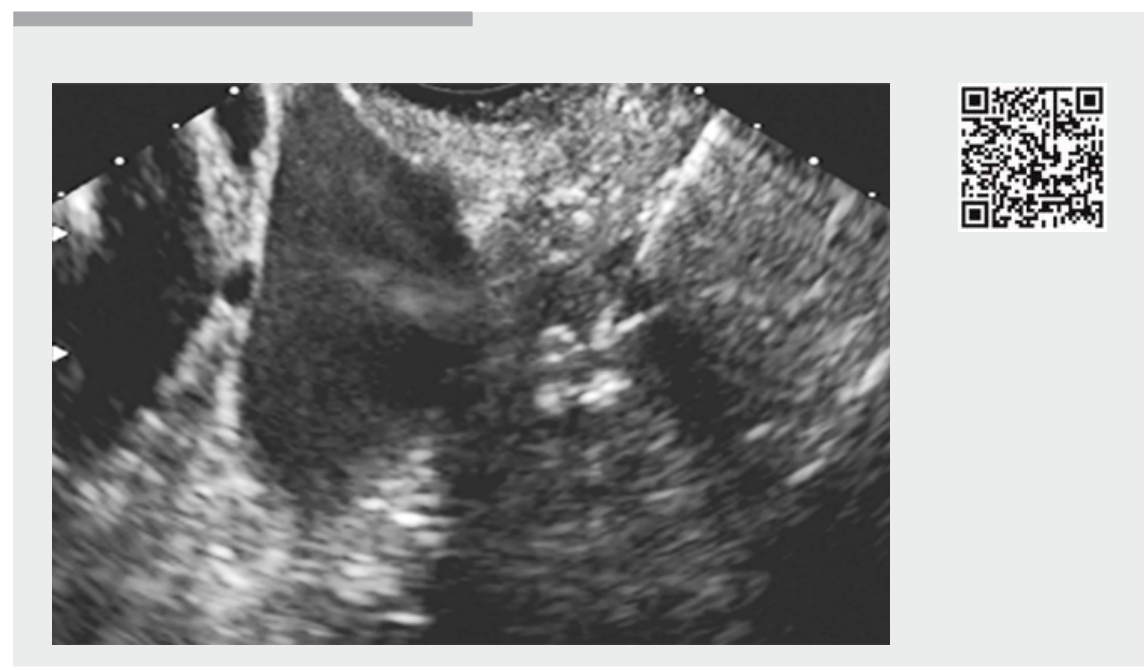

Video 1 Endoscopic ultrasound-guided fine needle injection (EUS-FNI) of 99\% ethanol into an insulinoma.

vein thrombosis was detected on EUS after the alcohol injection ( $>$ Video 1 ) and there was no evidence of acute pancreatitis after 2 days of in-hospital observation. At follow-up 2 weeks after discharge, the patient's serum glucose had returned to normal levels, she had lost 4 $\mathrm{kg}$ and the diazoxide dose was reduced. Pancreatic neuroendocrine tumors (pNETs) account for $1 \%-2 \%$ of all pancreatic tumors. PNETs are classified as either functional or nonfunctional, the latter being more common (up to $85 \%$ ). Among functional pNETs, insulinomas are the most common [1 - 2]. EUS is a useful diagnostic tool, showing a sensitivity of up to $85 \%$ [3]. Insulinoma resection was first reported in 1929 and remains the treatment of choice [4]. Nevertheless, EUS-FNI of ethanol into an insulinoma has been described as an efficient and safe technique for hypoglycemia resolution, and is indicated for patients with a prohibitive surgical risk [5].

Endoscopy_UCTN_Code_TTT_1AR_2AI

\section{Acknowledgments}

We must acknowledge Prof. Adhemar Monteiro Pacheco Júnior, MD, MSc, PhD, assistant physician with the Pancreas and Biliary Tract group of the Surgery Department, Santa Casa de São Paulo, for all the support the Surgery Department has given us, which has been indispensable for our good practice.

\section{Competing interests}

None

The authors

Flávio Amaro Oliveira Bitar Silva', Rogério Colaiacovo ${ }^{1}$, Osvaldo Araki ${ }^{1}$, Anna Fernanda Domene ${ }^{1}$, José Viana Lima Junior ${ }^{2}$, André de Moricz ${ }^{3}$, Lucio Rossini ${ }^{1}$

1 French-Brazilian Center of Echoendoscopy, Santa Casa de São Paulo, SP, Brazil

2 Endocrinology Department, Santa Casa de São Paulo, SP, Brazil

3 Department of Surgery, Santa Casa de São Paulo, SP, Brazil 

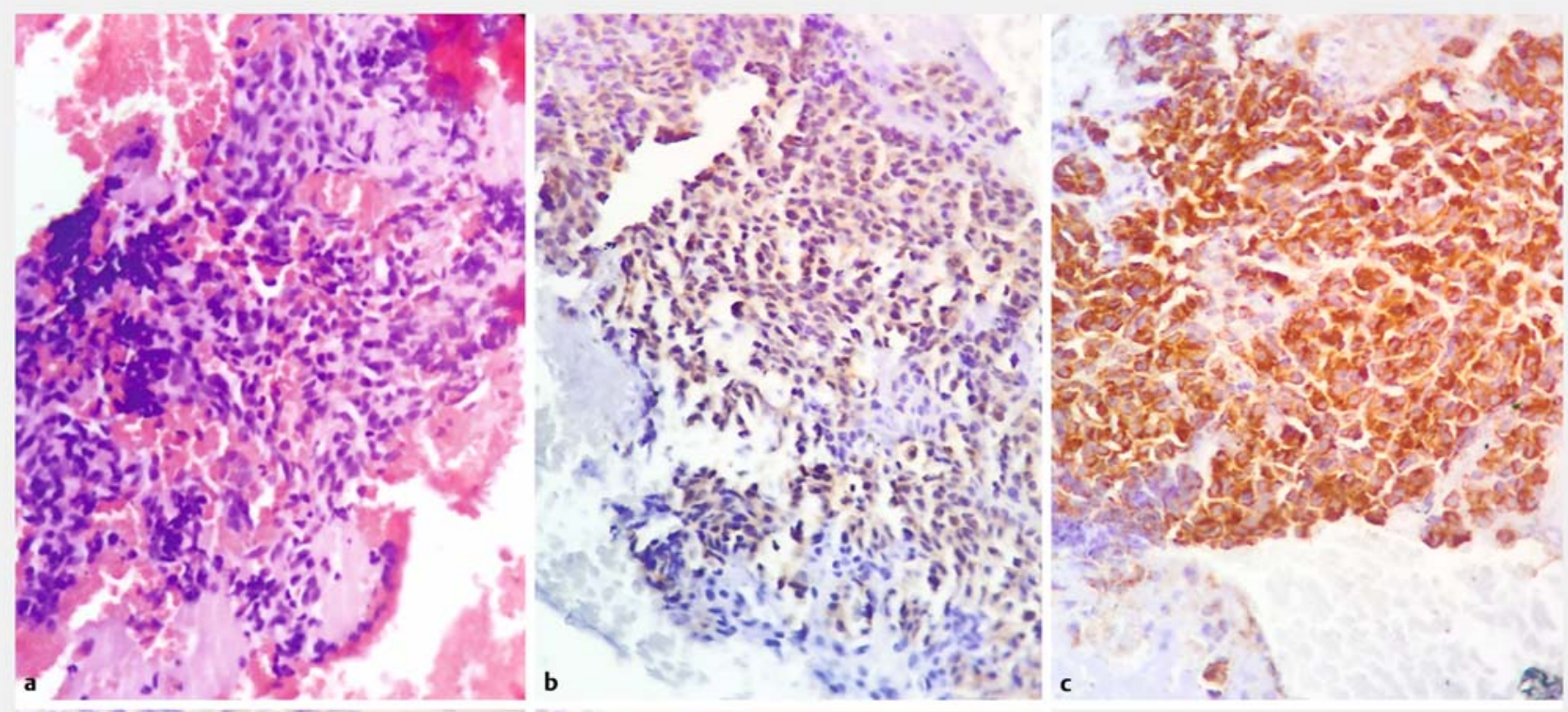

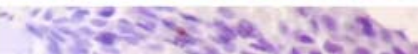
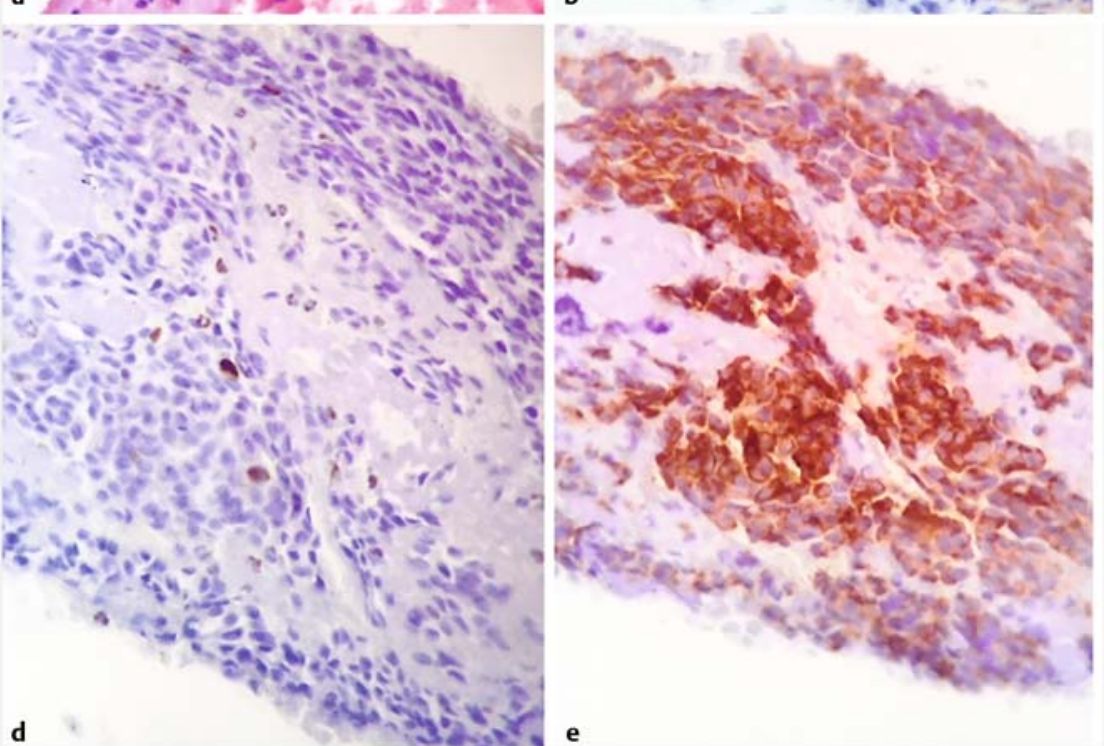

- Fig. 2 Histological analysis of the fine needle aspirate showing the appearance on: a hematoxylin and eosin (H\&E) staining; $\mathbf{b}$-e immunohistochemical staining with: b CD 56; c insulin; d Ki 67; e synaptophysin.

Corresponding author

\section{Flávio Amaro Oliveira Bitar Silva, MD}

Rua Cesário Mota Júnior, nº 112, no $4^{\circ}$ andar do Pavilhão Conde de Lara, Vila Buarque, São Paulo, SP, 01221-020, Brazil

flavioamaro.obs@gmail.com

References

[1] Dasari A, Shen C, Halperin D et al. Trends in the incidence, prevalence, and survival outcomes in patients with neuroendocrine tu- mors in the United States. JAMA Oncol 2017; 3: $1335-1342$

[2] Kasumova GG, Tabatabaie O, Eskander MF et al. National rise of primary pancreatic carcinoid tumors: comparison to functional and nonfunctional pancreatic neuroendocrine tumors. J Am Coll Surg 2017; 224: 1057 1064

[3] Placzkowski KA, Vella A, Thompson GB et al. Secular trends in the presentation and management of functioning insulinoma at the Mayo Clinic, 1987-2007. J Clin Endocrinol Metab 2009; 94: 1069-1073

[4] Howland G, Campbell WR, Maltby EJ et al. Dysinsulinism: Convulsions and coma due to islet cell tumor of the pancreas, with opera- tion and cure. Am Med Assoc 1929; 93 : 674-679

[5] Levy M], Thompson GB, Topazian MD et al. US-guided ethanol ablation of insulinomas: a new treatment option. Gastrointest Endosc 2012; 75: 200-206

\section{Bibliography}

DOI https://doi.org/10.1055/a-0820-1053

Published online: 11.1.2019

Endoscopy 2019; 51: E57-E58

(c) Georg Thieme Verlag KG

Stuttgart · New York

ISSN 0013-726X 